\title{
Pentetate Calcium
}

National Cancer Institute

\section{Source}

National Cancer Institute. Pentetate Calcium. NCI Thesaurus. Code C120569.

The calcium salt form of diethylene triamine pentaacetate (Ca-DTPA or pentetate calcium) with chelating activity. Upon administration, Ca-DTPA loses the Ca ion to form stable chelates with metal ions because DTPA has a higher affinity for heavy metal ions than for Ca ions. Specifically, this agent is able to bind to and form strong complexes with radioactive plutonium, americium, and cerium after internal contamination. The formation of these heavy metal chelates enhances radionuclide excretion. 